\section{P89 BECOMING A SEX WORKER: THE NEXUS BETWEEN VIOLENCE, GENDER DISADVANTAGE AND POVERTY}

doi:10.1136/sextrans-2012-050601c.89

M Shahmanesh,* S Wayal, F M Cowan. University College London, London, UK

Background Female sex workers (FSWs) in India remain most vulnerable to contracting HIV immediately after initiation into sex work. We explored the process of initiation to inform interventions. Methods In-depth interviews were conducted with sixteen purposively selected FSWs in Goa (December 2004-December 2005). The life narratives were interrogated using grounded theory.

Results The narratives showed a dynamic interplay between underlying vulnerabilities, precipitating factors, and how women entered sex work: The ubiquitous mitigating theme that emerged was violence: dysfunctional and violent family life; sexual violence; and violence from intimate male partners. Other underlying vulnerabilities were also manifestations of gender disadvantage: being unwanted; sexual naïveté; early sexual debut; entrapment in loveless marriages; and lack of life skills. Loss of social support through bereavement and abandonment or financial need, were the commonest events that precipitated entry into sex work. The clearest division in the route into sex work was between traditional caste-based sex workers (devadassi) and those who were either introduced by peers, or sold through a broker; however the underlying and precipitating factors for both routes were remarkably similar.

Conclusion The interplay between caste, economy, gender, and violence drives entry into sex work. HIV prevention interventions must work upstream on the context within which women enter sex work and downstream to strengthen their agency. The peers who introduce women into sex work are potential vehicles to "deliver" HIV prevention services. Ultimately, the challenge is to ensure that the structural vulnerabilities that prevent effective HIV prevention early after entry into sex work do not also become barriers preventing FSWs from accessing HIV treatment.

\section{P90}

\section{IS A $100 \%$ ANTENATAL HIV TESTING POSSIBLE?}

doi:10.1136/sextrans-2012-050601c.90

D Tegally, T Doucas, H Green, A Apoola.* Derby Hospitals NHS Foundation Trust, Derby, UK

Background Antenatal HIV testing in the UK has been a resounding success and is credited with reducing the rate of mother to child HIV transmission.

Aims To explore the characteristics of women who did not have the HIV test at booking, the reasons for declining and adherence to local policy on re-offering tests later in pregnancy. Between April 2010 and April 2011 a review of maternity case notes was carried out.

Results 6723 women were booked in early pregnancy in the relevant time period and $33(0.5 \%)$ of these women did not have a documented HIV test. Notes were only available for 32 of these women.

$31 / 32(96.8 \%)$ of the women were UK born, 27 (84.4\%) partners were UK born. 11 (34.4\%) women were pregnant for the first time. $13(40.6 \%)$ women had no documentation of a HIV test within a year of booking. There was no documentation of intravenous drug use in any of the women or their partners. 7 (21.9\%) women did not have any other blood borne virus testing done and none of these women had a recent documented HIV test either. Only in $8(25 \%)$ women was there documentation of reasons for declining. $5(12.5 \%)$ women were re-offered screening at about 28 weeks gestation in line with local policy with 4 (80\%) accepting. 6 of 18 women were offered testing on admission to the labour ward and 2 (33.3\%) were tested.
Discussion The maternity unit has a policy for dealing with women declining HIV testing in pregnancy and achieved a 99.6\% acceptance rate. This study shows that there are some problems with documentation of previous testing, risk assessment and consistently reoffering of the screening tests later on in pregnancy. When the test was re-offered later on in pregnancy there was a high acceptance rate emphasising the benefit of this approach. In view of these results further training of midwives with an emphasis on HIV risk assessment and re-offering testing later on in pregnancy will be implemented.

\section{P91 CARE OF HIV + WOMEN AGED 50 AND OVER: CAN WE DO BETTER?}

doi:10.1136/sextrans-2012-050601c.91

I Samuel, * J Welch, M Tenant-Flowers, M Poulton, C Taylor. King's College Hospital NHS Trust, London, UK

Background The prevalence of ageing HIV infected females continues to increase due to greater survival, delayed diagnosis and new infections; management of specific issues in this group becomes more challenging.

Aims/Objective To identify the specific care issues for women at and above 50 years.

Method Female patients aged 50 and over were identified from a female cohort of 828 and data collected retrospectively from case notes and electronic patient records.

Results and Discussion The sample consisted of 124 (15\% of 828) women, mean age 52 y, range $50-75$ y; $83 \%$ black ethnicity. $40.3 \%$ diagnosed at $50 \mathrm{y}$ or over. CD4 counts, mean 217 cells/ $\mu 1$ indicating $71 \%$ late diagnoses and $42 \%$ with advanced/very advanced HIV. $22.6 \%$ of the sample had attended hospital before and missed the opportunities for early diagnosis suggesting wider testing even in elderly. $4.5 \%$ women had premature menopause compared to $<1 \%$ in the general population, $7.1 \%$ early menopause, $28 \%$ had menopausal symptoms, $10 \%$ had HRT. On opportunistic testing of BMD tests, 5/8 women had evidence of osteoporosis/osteopenia. $54 \%$ of the whole group had vitamin D deficiency. $38 \%$ had abnormal cervical cytology, of which $16.5 \%$ were high grade. A high degree of obesity (48\%) and other co-morbidities (90\%) identified.

Conclusions Special care for premature/early menopause including early counselling regarding reproductive options, wider HIV testing regardless of age, screening for osteoporosis, breast and cervical cancer, holistic approach in the management of obesity and other co-morbidities. Consideration needed as to how care is provided for this group within current health care settings.

\section{P92 ROUTINE ENQUIRY FOR DOMESTIC VIOLENCE, ALCOHOL AND DRUG USE IN A SEXUAL HEALTH CLINIC}

doi:10.1136/sextrans-2012-050601c.92

W C Loke, ${ }^{*}$ J Maclellan, J Maurice. Barnet Enfield Haringey Mental Health Trust

Background Addressing domestic violence (DV) against women is highlighted in the 2011/12 NHS Operating Framework. Genitourinary medicine is cited as one of the services where victims of abuse may present. Alcohol and drug use are recognised as factors affecting sexual risk taking behaviour. Routine enquiry for DV (women only) and drug and alcohol use was implemented in this service in June 2011.

Aims To audit if routine enquiry for DV, alcohol and drug use was done and identify interventions when DV was disclosed or if risk taking was found to be affected by drug or alcohol use. 
Methods A casenote review was done on all new and rebook (attending again after 3 months or more for a new episode of care) patients who attended during the first week of September 2011. The history proforma included questions on DV, alcohol consumption, recreational drug use and if they felt this affected their risk taking. Asymptomatic patients for screening were excluded as no detailed history was taken.

Results The history proforma was filled out for 55 women and 22 men. All were heterosexuals. The median age was 25. 26 attended for contraception, 44 for sexual health screen and 7 for both. 21 were rebook patients. 38/55 (69\%) women were asked about DV. Five gave a history of DV, four were historic, one unspecified. 63 $(82 \%)$ and $62(81 \%)$ were asked their alcohol and drug use respectively. One man disclosed drinking $>21$ units/week and that it affected his risk taking. 10 used drugs: six cannabis, one cocaine and three unspecified. None were referred to other agencies. Of those not asked about DV, alcohol or drug use, no reason was documented.

Discussion The relatively low rate of enquiry for DV may increase with training and awareness raising. Only one patient considered his risk taking affected by alcohol. This may be due to the lack of sensitivity of direct questioning. Closer working with supporting agencies for DV, alcohol and drug use may increase referrals.

\section{P93 THE ACCEPTABILITY OF ROUTINE ENQUIRY OF GENDER- BASED VIOLENCE AT SEXUAL HEALTH CLINICS}

\section{doi:10.1136/sextrans-2012-050601c.93}

K Innes, P Fraser, S D K Baguley. NHS Grampian Sexual Health Service

Background In 2008/09, 53861 incidents of GBV were reported to the police in Scotland; $84 \%$ were male perpetrator against female victim and $14 \%$ female vs male. National guidance recommends routine enquiry of abuse (REA) amongst female attendees at sexual health clinics. Section SH 4.3 of the Sexual Health and Blood Borne Virus Framework says that levels of GBV should be recorded. Research in an antenatal setting found that $99 \%$ of respondents thought REA was acceptable. However, a literature review found no assessment of the acceptability of REA in sexual health clinics. It is also unclear what value there may be of REA in male attendees.

Aim In order to elucidate these areas, attendees at the sexual health service in the city were asked for their opinion of REA and their experience of GBV.

Method As part of our 2010 patient satisfaction survey, attendees were asked: We are considering asking people whether they have been a victim of domestic violence (eg, being hit by a girlfriend, boyfriend, husband or wife). Q1. Do you think we should routinely ask patients this? Q2. Have you ever been the victim of domestic violence? (Support is available here)

Results Of the 110 respondents, 100 answered Q1 and 104 Q2. Of the $100,81 \%$ (95\% CI $72 \%$ to $88 \%$ ) said REA would be acceptable. No significant difference between men and women or abused and non-abused ( $9 / 10$ of abused, $90 \%$ (CI $56 \%$ to $100 \%))$. One man (1/42 $2 \%$ (CI $0.1 \%$ to $12.6 \%$ ) and 10 women $(10 / 42,16 \%$ (CI $8 \%$ to $28 \%$ ) reported GBV ( $p=0.025$ for gender difference). One man and three women declined to answer. Comments from women who reported GBV included "Other departments should deal with this " $-38 \mathrm{yr}$ old seen at CSRH and "Gives them someone who will listen" - $64 \mathrm{yr}$ old seen at GUM.

Discussion REA was acceptable to the majority. GBV history was not insignificant in men. This survey used a narrow definition of GBV and reports may have been higher had a more inclusive definition been used. Implications are that REA should be done in clinics and should be considered in men, perhaps using a self-completed questionnaire.

\section{P94 \\ PREVALENCE AND CORRELATES OF SEXUAL VIOLENCE IN FEMALE ATTENDEES AT GENITOURINARY MEDICINE CLINICS}

doi:10.1136/sextrans-2012-050601c.94

${ }^{1} \mathrm{~J}$ White, ${ }^{2} \mathrm{~N}$ Biros, ${ }^{2} \mathrm{D}$ Holland. ${ }^{1}$ Genitourinary Medicine, Guy's and St Thomas' NHS Trust, c/Lydia Clinic, St Thomas' Hospital, London, UK; ${ }^{2}$ Guy's and St Thomas' NHS Trust, London, UK

Background Sexual violence (SV) is common but under-reported in the UK. Victims of SV may be more likely to attend genitourinary medicine (GUM) clinics but there are no recent urban data.

Aims To determine the prevalence and correlates of SV in female GUM attendees. To assess whether routine enquiry on SV is warranted and to gauge if specific SV resources are needed in GUM. Methods Questionnaire-based survey offered to all women attending our two urban walk-in GUM clinics. Participants selfcompleted anonymised proformas about any experiences of SV using a broad definition of SV. Demographic, clinical and behavioural data were also collected.

Results Analysis of the initial 164 surveys showed a median age of 27 (IOR 23-31). Ethnicity was typical of the clinics' populations: $62 \%$ UK born; 40\% White British, 21\% White other, 16\% Black British, 8\% Black African and 7\% Black Caribbean. When asked about a history of SV ever, $17 \%$ responded yes; in $36 \%$ of these women SV had happened more than once. Median age at the time of SV was 19 years (range 6-40); 22/164 (13\%) described the SV as rape/sexual assault and in two women this was in the last year. Only two women described their assailant as a stranger; one confirmed her SV was gang-related. Additional women responded to queries on forced oral sex $5 \%$, forced touching $7 \%$, forced sex without a condom $11 \%$. Of 32 women who told someone it included a health professional in only $28 \%$ and the police in $25 \%$. $78 \%$ of all participants agreed it was helpful to ask routinely about SV and $87 \%$ felt that a SV worker was needed in the clinic; four women indicated that their reason for attending GUM today was SV-related. Qualitative comments were mostly positive and accepting of SV enquiry, though several women expressed negative comments about the difficult emotions it had evoked.

Conclusions Women attending GUM have a high prevalence of SV. Further study is warranted within GUM settings to establish if routine enquiry and SV service provision should be core business.

\section{P95 ATTITUDES TO SEXUAL HISTORY TAKING AND STI SCREENING IN A NON-SEXUAL HEALTH DELIVERED HIV SERVICE}

doi:10.1136/sextrans-2012-050601c.95

${ }^{1}$ E F C Salter, * ${ }^{2} \mathrm{E}$ Magee, ${ }^{2} \mathrm{~S}$ L Allstaff. ${ }^{1}$ University of Dundee Medical School, Dundee, UK; ${ }^{2}$ NHS Tayside, Dundee, UK

Background An audit within our HIV service failed to meet national sexual health targets. Barriers to sexual history ( $\mathrm{SHx}$ ) taking and STI screening include fear of causing offence. This may be more so in HIV services delivered by non-sexual health clinicians.

Aim To determine patients' attitudes to SHx taking and STI screening in an infectious diseases-led HIV service.

Methods An anonymous, semi-structured questionnaire was offered to all clinic attendees from September-December 2011. Data collected included demographics, HIV history, sexual history and attitudes to SHx taking and STI screening.

Results Of the 101 questionnaires returned; 68\% were male and almost two thirds were aged 26-40. 30\% were MSM. 35\% were in sero-discordant regular relationships. $37 \%$ had one partner in the 


\section{Corrections}

Loke WC, Maclellan J, Maurice J. Abstract P92 Routine enquiry for domestic violence, alcohol and drug use in a sexual health clinic. Sex Transm Infect 2012;88:A40-A41. The third author, Emma Jones, was omitted from the original author list. She is listed at the affiliation Barnet, Enfield and Haringey Mental Health NHS Trust.

Sex Transm Infect 2012;88:474. doi:10.1136/sextrans-2012-050601c.92corr1 